\title{
Mid-infrared coronagraph for SPICA
}

\author{
K. Enya ${ }^{a^{*}}$, L. Abe ${ }^{b}$, K. Haze ${ }^{a}$, S. Tanaka ${ }^{a}$, T. Nakagawa ${ }^{a}$, H. Kataza ${ }^{a}$, S. Higuchi ${ }^{a}$,

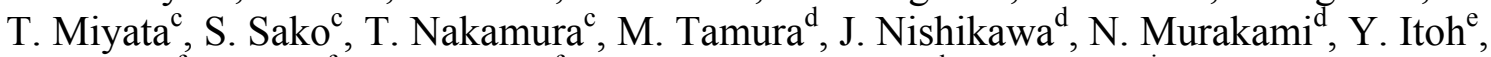

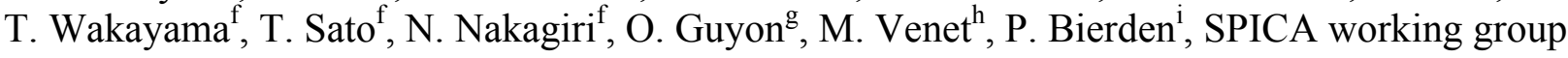 \\ ${ }^{a}$ Department of Infrared Astrophysics, Institute of Space and Astronautical Science, \\ Japan Aerospace Exploration Agency, Yoshinocai 3-1-1, Sagamihara, Kanagawa 229-8510, Jpapan \\ ${ }^{\mathrm{b}}$ Laboratoire Hippolyte FIZEAU, UMR-6525 CNRS, 28, avenue Valrose, 06108 Nice, France \\ 'Institute of Astronomy, University of Tokyo, 2-21-1, Osawa, Mitaka, Tokyo, 181-0015, Japan \\ ${ }^{\mathrm{d}}$ National Astronomical Observatory of Japan, 2-21-1, Osawa, Mitaka, Tokyo, 181-8588, Japan \\ ${ }^{e}$ Laboratory of Solar System Physics, Graduate Shool of Science and Technology, Kobe University, \\ 1-1, Rokkodai-cho, Nada, Kobe 657-8501, Japan \\ ${ }^{\mathrm{f}}$ Nanotechnology Research Institute, Advanced Industrial Science and Technology, \\ Umezono 1-1-1, Tsukuba, Ibaraki 305-8568, Japan \\ ${ }^{\mathrm{g}}$ Subaru Telescope, National Astronomical Observatory of Japan, 650 N. A'ohoku Place, Hilo, \\ HI 96720, US \\ hobservatoire Astronomique de Marseille-Provence, Pôle de l'Étoile Site de Château-Gombert 38, \\ rue Frédéric Joliot-Curie, 13388 Marseille cedex 13, France \\ ${ }^{\mathrm{i}}$ Boston Micromachines Corporation, 30 Spinelli Place Cambridge, MA 02138, USA
}

\begin{abstract}
The SPace Infrared telescope for Cosmology and Astrophysics (SPICA) is a infrared space-borne telescope mission of the next generation following AKARI. SPICA will carry a telescope with a $3.5 \mathrm{~m}$ diameter monolithic primary mirror and the whole telescope will be cooled to $5 \mathrm{~K}$. SPICA is planned to be launched in 2017, into the sun-earth L2 libration halo orbit by an H II-A rocket and execute infrared observations at wavelengths mainly between 5 and 200 micron. The large telescope aperture, the simple pupil shape, the capability of infrared observations from space, and the early launch gives us with the SPICA mission a unique opportunity for coronagraphic observation. We have started development of a coronagraphic instrument for SPICA. The primary target of the SPICA coronagraph is direct observation of extra-solar Jovian planets. The main wavelengths of observation, the required contrast and the inner working angle (IWA) of the SPICA coronagraph are set to be 5-27 micron (3.5-5 micron is optional), $10^{-6}$, and a few $\lambda / D$ (and as small as possible), respectively, in which $\lambda$ is the observation wavelength and $D$ is the diameter of the telescope aperture (3.5m). For our laboratory demonstration, we focused first on a coronagraph with a binary shaped pupil mask as the primary candidate for SPICA because of its feasibility. In an experiment with a binary shaped pupil coronagraph with a He-Ne laser $(\lambda=632.8 \mathrm{~nm})$, the achieved raw contrast was $6.7 \times 10^{-8}$, derived from the average measured in the dark region without active wavefront control. On the other hand, a study of Phase Induced Amplitude Apodization (PIAA) was initiated in an attempt to achieve better performance, i.e., smaller IWA and higher throughput. A laboratory experiment was performed using a He-Ne laser with active wavefront control, and a raw contrast of $6.5 \times 10^{-7}$ was achieved. We also present recent progress made in the cryogenic active optics for SPICA. Prototypes of cryogenic deformable by Micro Electro Mechanical Systems (MEMS) techniques were developed and a first demonstration of the deformation of their surfaces was performed with liquid nitrogen cooling. Experiments with piezo-actuators for a cryogenic tip-tilt mirror are also ongoing.
\end{abstract}

Keywords: SPICA, infrared, coronagraph, exo-planet, deformable mirror, tip-tilt

* correspondance to SPICA coronagraph: enya@ir.isas.jaxa.jp

Space Telescopes and Instrumentation 2008: Optical, Infrared, and Millimeter, edited by Jacobus M. Oschmann, Jr., Mattheus W. M. de Graauw, Howard A. MacEwen, Proc. of SPIE Vol. 7010, 70102Z, (2008) · 0277-786X/08/\$18 - doi: 10.1117/12.788509

Proc. of SPIE Vol. $701070102 Z-1$ 

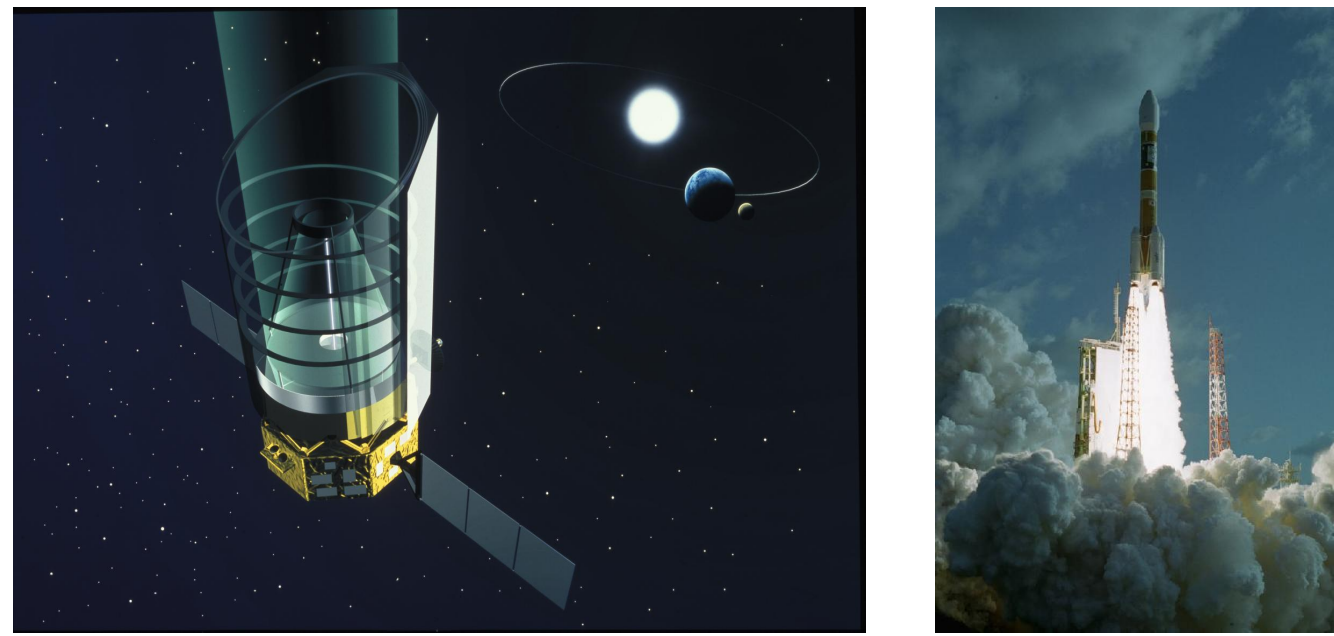

Figure 1. Left: Artist's view showing the SPICA telescope in orbit. Right: a HII-A rocket (C JAXA).

\section{INTRODUCTION}

We consider the direct observation of exo-planets to be one of the most important issues for space science in the near future. More than 250 exo-planets have been discovered by indirect observation of the Doppler shift of the spectrum or the variable luminosity of a star due to the transit of a planet ${ }^{[1,2,3]}$. However, such indirect observations do not usually provide the spectrum, luminosity or other important properties of the planet itself. Thus, a systematic study of extra-solar planets by direct observation is required, in which the enormous contrast between the central star and the planet is quite a serious problem. The typical contrast is $\sim 10^{-10}$ in the visible light region but is reduced to $\sim 10^{-6}$ in the mid-infrared ${ }^{[4]}$. With coronagraphs, which were first evaluated for solar observation ${ }^{[5]}$, the contrast between an extra-solar planet and its central star is expected to be reduced. Coronagraphic observations in space have an essential advantage over groundbased observations because they are completely free from air turbulence. Thus, infrared observation using a coronagraph and a space telescope could be one of the most feasible and therefore most promising ways to realize direct detection of exo-planets.

The SPace Infrared telescope for Cosmology and Astrophysics (SPICA) is one of the next generation of infrared spaceborne telescope projects ${ }^{[6,7]}$ following AKARI, which was launched in February $2006^{[8]}$. SPICA will carry an on-axis Ritchey-Chretien telescope which has a monolithic mirror with a $3.5 \mathrm{~m}$ aperture. The whole telescope is cooled to $5 \mathrm{~K}$ by radiation cooling and mechanical cryo-coolers. The required optical quality of the telescope is a diffraction limited image at a wavelength of 5 micron. The main observation wavelength is in the range 5-200 micron. SPICA gives us a unique opportunity for coronagraphic observation because of the mid-infrared observation capability, the large telescope aperture and simple pupil shape provided by the monolithic mirrors. Therefore, SPICA will be equipped, together with other general purpose instruments, with a coronagraph ${ }^{[9-12]}$. We have set the primary target of the SPICA coronagraph to be the direct observation of extra-solar Jovian planets. The specification of the SPICA coronagraph is shown in table 1. Figure 2 shows the distribution of the field of view for focal plane instruments on SPICA.

Special space missions targeting terrestrial extra-solar planets (e.g., TPF-C ${ }^{[13]}$ and related missions, TPF- ${ }^{[14]}$, DARWIN ${ }^{[15]}$, use of petal-shaped occulter ${ }^{[16]}$ ) have been proposed. These missions are quite huge and need ultimate performance to detect the terrestrial planets, and therefore launches for these missions are planned to be significantly later than the launch of SPICA. The James Webb Space Telescope (JWST) is a general purpose space telescope $\operatorname{mission}^{[17]}$, and is in the same generation as SPICA. It will have coronagraphic instruments; however, the primary mirror of JWST consists of many segments which limit the coronagraphic performance. Furthermore, the SPICA coronagraph will have a spectroscopic mode which JWST will not have. From direct observation of Jovian-type extra-solar planets by high quality coronagraphic imaging and spectroscopy in the SPICA mission, we expect to characterize extra-solar planetary systems and reveal their formation history 

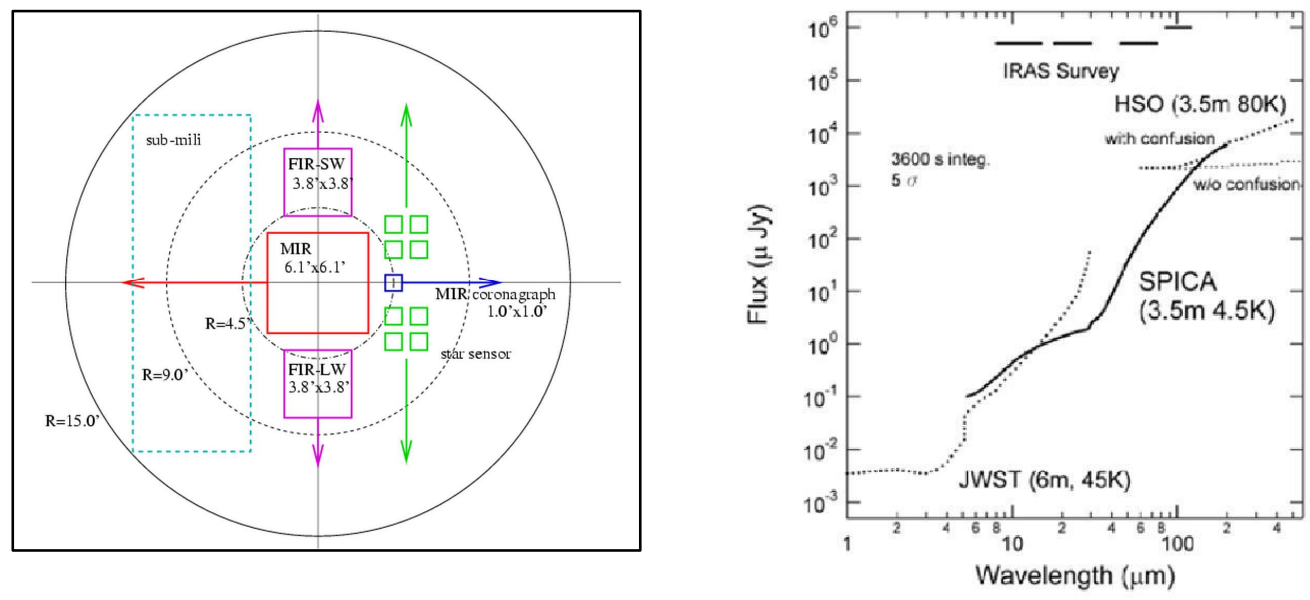

Figure 2. Left: distribution of the focal plane for the expected instrumentation. Right: sensitivity of SPICA telescope

Table 1. Specification of the SPICA coronagraph instrument.

\begin{tabular}{ll}
\hline \hline Parameter & Specification \\
\hline Core wavelength $(\lambda)$ & $5-27$ micron (3.5-5 micron is optional) \\
Observation mode & Imaging, Spectroscopy \\
Coronagraphic method & binary shaped pupil mask, \\
& and/or hybrid (binary shaped pupil + PIAA) \\
& $\sim 3.5 \times \lambda / D^{*}$ (binary shaped pupil mask mode) \\
Inner working angle (IWA) & $<2 \times \lambda / D^{*}$ (hybrid mode) \\
& $\sim 30 \%$ (binary shaped pupil mask mode) \\
Throughput & $\sim 80 \%$ (hybrid mode) \\
& $\sim 30 \times \lambda / D$ (binary shaped pupil mask mode) \\
Outer working angle (OWA) & $\sim 10 \times \lambda / D$ (hybrid mode) \\
& $\geq 10^{6}$ \\
Contrast between IWA and OWA & $1 \mathrm{k} \times 1 \mathrm{k}$ format Si:As array, $0 . " 1 /$ pixel \\
Detector & $1 \times 1$, \\
Field of View & $<$ IWA \\
Spatial resolution & $\sim 200$ \\
Spectral resolution &
\end{tabular}

$* D=3.5 \mathrm{~m}$ is the entrance pupil diameter of the SPICA telescope 

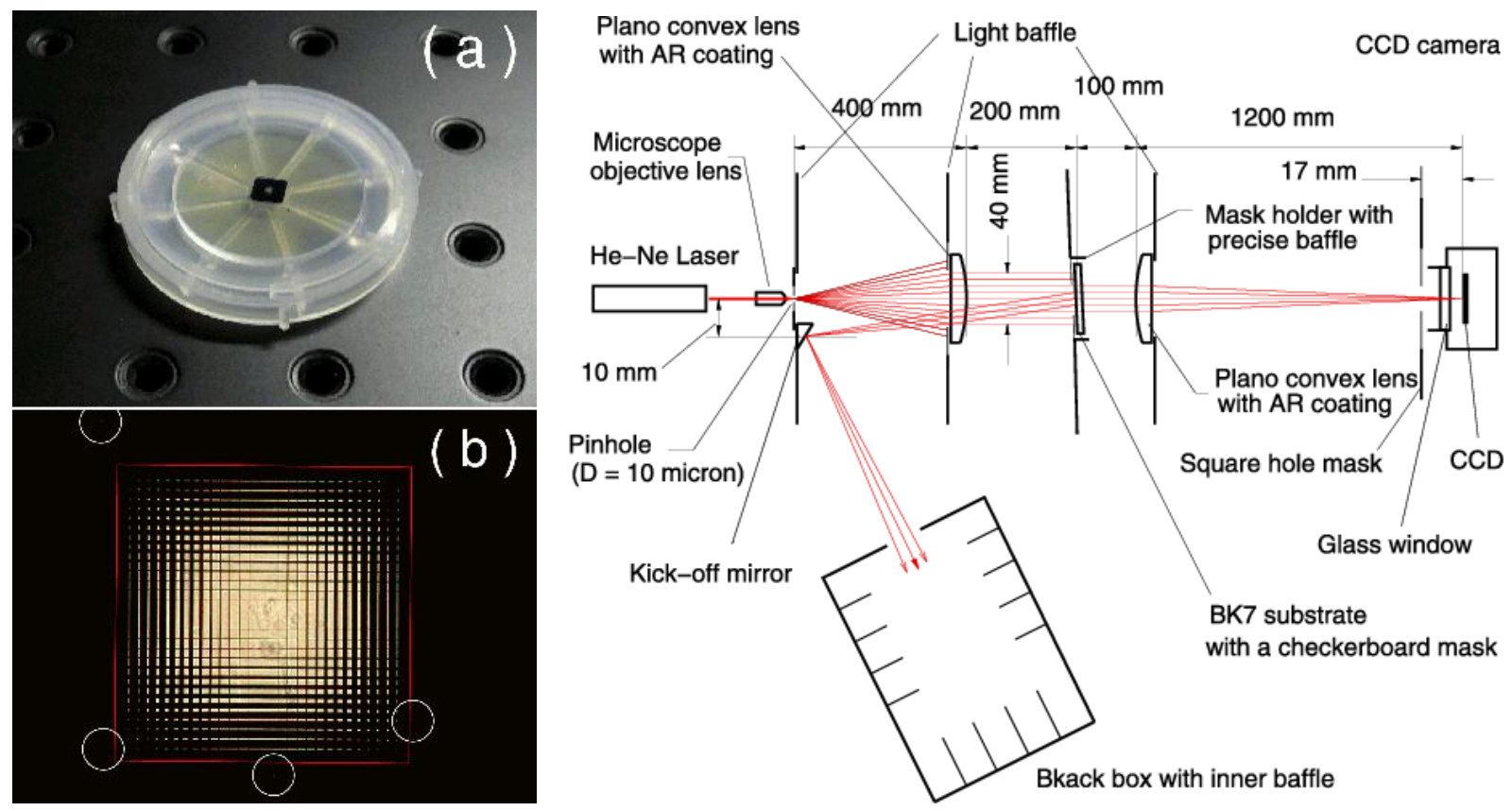

Figure 3. Top-left: manufactured mask \#3. Bottom-left: A photograph of mask \#3 by microscope. Right: Optical configuration of the experiment. All the optical devices were set on a table with air suspension in a dark room with an air cleaning system flowing from the top of the room onto the optical table.

\section{DEVELOPMENT OF THE CORONAGRAPH}

Recently, we succeeded in laboratory demonstrations of coronagraphs, in which the contrast requirement of the SPICA coronagraph $\left(10^{-6}\right)$ was satisfied using visible light, as described in the following sections. These are important steps toward the development of a MIR coronagraph in a cryogenic and vacuum environment.

\subsection{Experiment of a binary shaped pupil mask coronagraph}

In selecting a coronagraph for SPICA, some peculiar requirements had to be considered. The SPICA coronagraph had to work in the mid-infrared wavelength region in a cryogenic environment, so a coronagraph without transmissive devices was preferable. The coronagraph should not be subject to telescope pointing errors caused by vibration of the cryogenic cooling system and the attitude control system. Achromatism was also an important property. After taking these points into consideration, a coronagraph with a binary shaped pupil mask ${ }^{[18-25]}$ was selected as the primary target of our study and an experimental demonstration that this was a feasible solution was carried out.

We carried out an experiment to investigate and demonstrate the performance of a coronagraph with checkerboard masks using visible light in an air ambient. Of our 3 checkerboard masks, we briefly show an experiment using the last one (mask \#3) in this paper. For more details or the results using other masks, please refer to our other publications ${ }^{[26,27]}$. Binary masks consisting of $100 \mathrm{~nm}$ thick aluminum patterns on BK7 glass substrates were made by nano-fabrication technology using electron beam patterning and a lift-off process. Optimization of the checkerboard mask was executed using LOCO software presented by Vanderbei $(1999)^{[28]}$. The contrast, IWA and OWA of mask \#3 were designed to be $10^{-10}, 3.7$ and $35 \lambda / D$, respectively. Anti reflection (AR) coatings were applied to both sides of the mask. The left panel of figure 3 shows the manufactured mask \#3. The configuration of the experiment is shown in the right panel of figure 3. The setup of the experiment was in a dark room with an air cleaning system employing HEPA filters and the optical 

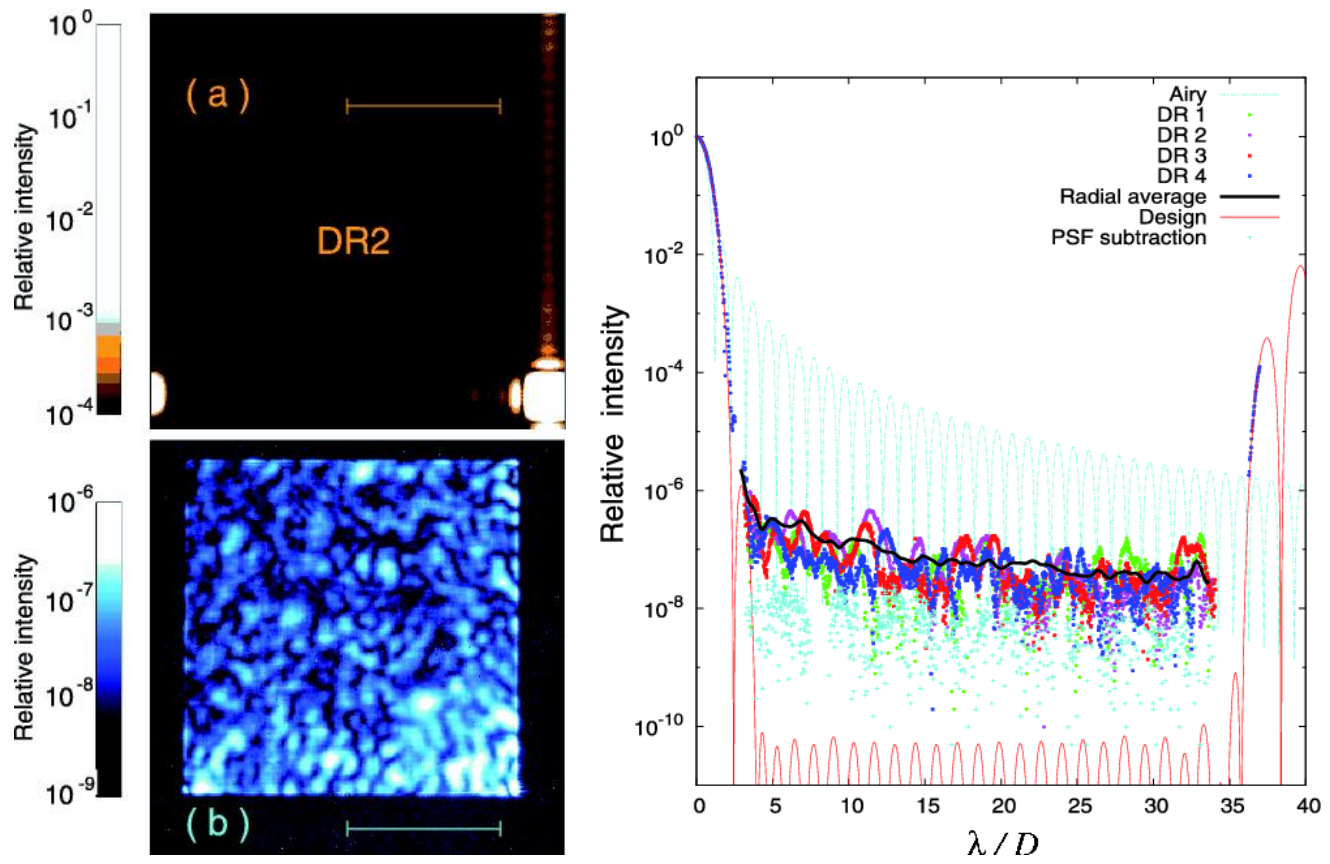

Figure 4. Left: observed coronagraphic images. The top and the bottom panels show a low sensitivity image of the whole PSF and a high sensitivity image of the dark region in the PSF through a square hole mask. Right: observed and theoretical profiles are shown as well as the theoretical Airy profile. Each profile is normalized by the peak intensity in each image.

devices were set on a table with air suspension. A He-Ne laser with a 0.63 micron wavelength was used as the light source. A spatial filter, consisting of a microscope objective lens and a 10 micron diameter pinhole, was used. Collimation was done using a plano-convex lens with an AR coating and the collimated beam was incident on the pupil mask. After passing through the mask, the beam was focused by a lens with the same specification as the collimator lens but with a longer focal length as shown in figure 3. A cooled CCD camera was used for imaging. Wavefront control by adaptive optics was not applied. We used several different exposure times to expand the dynamic range of the measurement. For measurement of the dark region, a 20 micron thick black coated SUS304 mask was set in front of the CCD camera to reduce the scattered light in the camera.

The left panels of figure 4 show the observed point spread function (PSF) with the checkerboard mask coronagraph, and a profile of this PSF is shown in the right panel of figure 4. The profile of the observed PSF at the core in which the relative intensity is $>10^{5}$ is identical to the theoretical one. The contrast derived by averaging in the dark region using a linear scale is $6.7 \times 10^{-8}$. Both the IWA and OWA obtained from the experiment are also consistent with the designed values. Therefore, it was concluded that the coronagraph with a binary checkerboard pupil mask with or without a large central obstruction works at the target contrast $\left(10^{-6}\right)$. We also concluded that speckle is the primary limiting factor of the coronagraphic performance in this experiment.

\subsection{Experimental study of PIAA coronagraph}

On the other hand, following the development of the coronagraph with a binary shaped pupil mask, the study of a coronagraph with phase-induced amplitude apodization (PIAA) using two mirrors ${ }^{[29,30]}$ was also started in an attempt to achieve higher performance. In principle the PIAA coronagraph provides a smaller IWA $(\sim 1 / 2$ or less than the IWA of the binary shaped mask ) and a higher throughput ( 100\%), though its development is more challenging.

The high throughput and small IWA of the PIAA coronagraph are achieved without it being affected too much by resolved stellar disks or telescope tip-tilt errors. However, a PIAA designed to give high contrast by itself would require optical shapes that are difficult to polish, as well as a reduced bandwidth because of chromatic diffraction. These problems can be simultaneously solved by adopting a hybrid PIAA design (Pluzhnik et al. $2006^{[31]}$ ), where the 

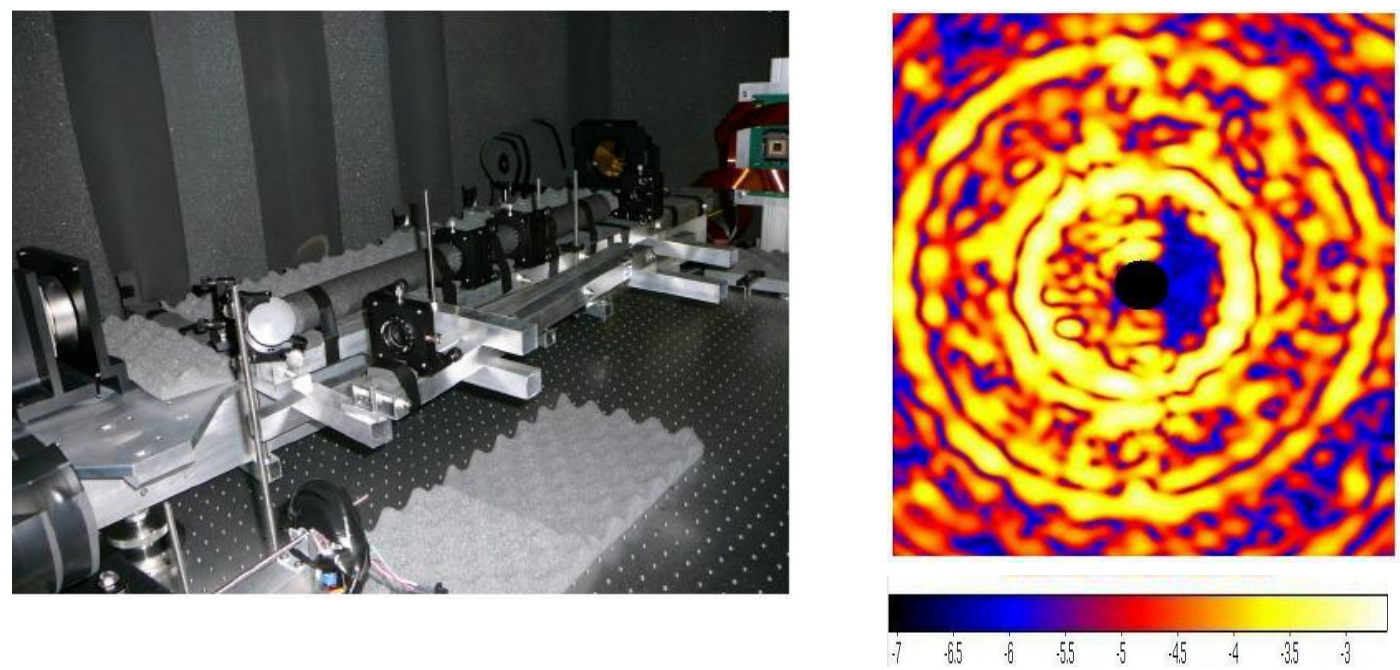

Figure 5. Left: optical configuration used for the laboratory demonstration of PIAA. Right: observed coronagraphic image shown on a $\log$ scale (Tanaka, Guyon et al. 200 ${ }^{[32]}$ obtained in the laboratory of Guyon et al.). The core of the PSF is obstructed by a focal plane mask in which the radius is $1.5 \lambda / D$. The dark region at the right of the core is produced by wavefront control using a deformable mirror with $32 \times 32$ actuators. The average contrast of the dark region is $6.5 \times 10^{-7}$.

apodization created by the two-mirror system is moderated by combining a classical apodizer with PIAA apodization. Tanaka et al. (2007) ${ }^{[32]}$ proposed the implementation of a hybrid PIAA system with properly designed binary apodization masks, and showed in the laboratory that such a combination is a robust approach to high contrast imaging. A laboratory experiment was performed using a He-Ne laser in air as shown in figure 5. Active wavefront control was applied using a Micro Electro Mechanical Systems (MEMS) deformable mirror with $32 \times 32$ channels provided by Boston Micromachines Corp. (BMC). The whole of the optics for the experiment was set in a clean room. Thanks to thermal stabilization and the mechanical isolation of the whole PIAA optics, the image drift on the final focus was kept quite small ( $\sim$ a few pixels in 1-2 hours). With this stability, a standard speckle nulling technique was used to successfully eliminate broad speckles in half the image plane. In an experiment developed by Guyon and his colleagues at the laboratory in the SUBARU observatory, a contrast of $6.5 \times 10^{-7}$ at a separation of $\sim 1.5 \mathrm{~N} / \mathrm{D}$ from the center of the PSF was obtained (Tanaka et al. $2007^{[32]}$ ).

\subsection{Other products}

Before the final design decisions for the coronagraphic instrument are made and demonstration of a MIR coronagraph in a cryogenic and vacuum environment is completed, there are some other projects relating to the SPICA coronagraph being undertaken.

- A multi-stage Prolate Apodized Lyot Coronagraph (MS-PALC) $)^{[33]}$, a variation of the classical Lyot type coronagraph, has also been experimentally studied as reported by Abe et al. (2008, this conference) ${ }^{[34]}$, Venet $(2006)^{[35]}$. This has the potential to provide a small IWA $(1 \sim 1.5 \lambda / D)$.

- Manufacturing tests of binary shaped masks for the MIR coronagraph are on-going. These masks are $\sim 1 \mathrm{~cm}$ size, i.e., larger than the masks shown in sec. 2.1, and some of them are intended to be free standing devices, i.e., have no substrate.

- We are developing a vacuum chamber, in which the most important goal is demonstration of a MIR coronagraph in a vacuum and cryogenic environment; however, we think the chamber has the potential to enable us to improve the performance of our experiment with visible light. 

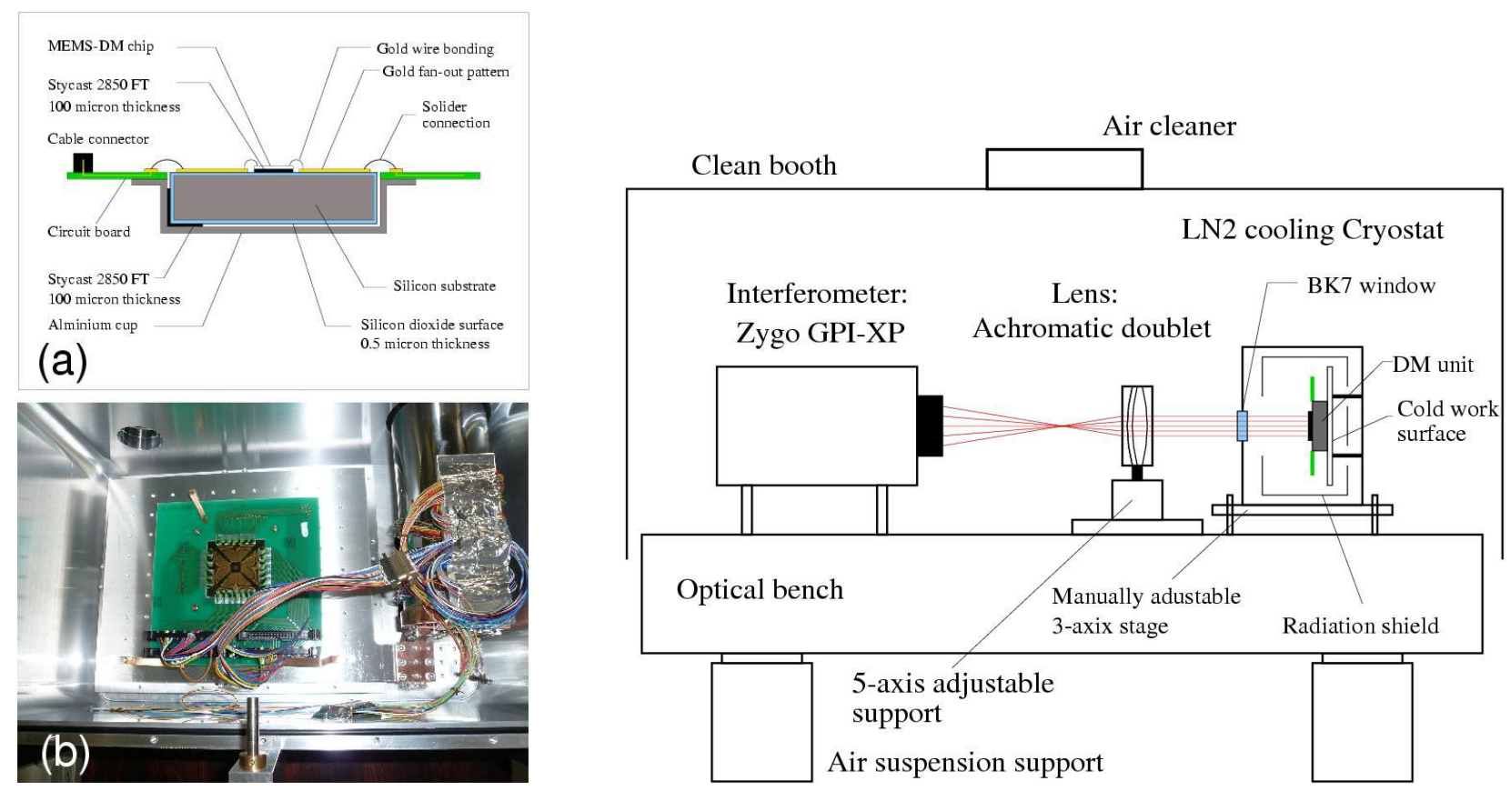

Figure 6: Top left: schematic view of the prototype DM unit. Bottom left: The DM unit installed in a cryostat. The radiation shield and temperature sensor are not shown in this photograph. Right: measurement configuration.

\section{ACTIVE OPTICS}

\subsection{Cryogenic deformable mirror}

The specification of the image quality of the SPICA telescope is 5micron diffraction limit, corresponding to a wavefront error of $350 \mathrm{~nm} \mathrm{rms}$, which is insufficient for the coronagraphic instrument. Therefore we need active optics to compensate for the wavefront error in the SPICA telescope, like a deformable mirror (DM) which can be operated at cryogenic temperatures ${ }^{[36,37]}$. One of the usual types of DM is based on the piezoelectric effect; however, this effect tends to be smaller at cryogenic temperatures (the piezoelectric coefficient at $5 \mathrm{~K}$ is $\sim 1 / 10$ of that at ambient temperature: see also Mulvihill et al.(2003) ${ }^{[37]}$. Recently, DMs fabricated using Micro Electro Mechanical Systems (MEMS) techniques have been developed ${ }^{[38,39]}$. Operation of the MEMS DM is based on Coulomb forces, so does not depend on the piezoelectric effect. The possibility of realizing large format and compact DMs is also an advantage of MEMS technology. For example, MEMS DMs with $32 \times 32$ channels are commercially available. However, the stress introduced into MEMS DMs by cooling due to mismatched coefficients of thermal expansion (CTE) can cause distortion and even destroy the mirror.

Hence, we proposed to develop and evaluate a prototype cryogenic DM system based on MEMS mounted on a special substrate designed to reduce thermal stress, as shown in figure 6 . The mirror was mounted on a silicon substrate in order to minimize distortion and prevent it from being permanently damaged by thermal stresses introduced by cooling. The silicon substrate was oxidized and had a metal fan-out pattern on the surface. A MEMS silicon DM chip with 32 channels was bonded to the substrate. For cryogenic tests, we constructed a measurement system consisting of a Fizeau interferometer, a cryostat cooled by liquid nitrogen, zooming optics, and electric drivers. The surface of the mirror at $95 \mathrm{~K}$ deformed in response to the application of a voltage, and no significant difference was found between the deformation at $95 \mathrm{~K}$ and that at room temperature as shown in figure 7 . The heat generated by the cryogenic DM was also measured, and we suggest that this is small enough for it to be used in a space cryogenic telescope. The properties of the DM remained unchanged after 5 cycles of vacuum pumping, cooling, heating, and venting. We conclude that fabricating cryogenic DMs employing MEMS technology is one of the promising approachs 


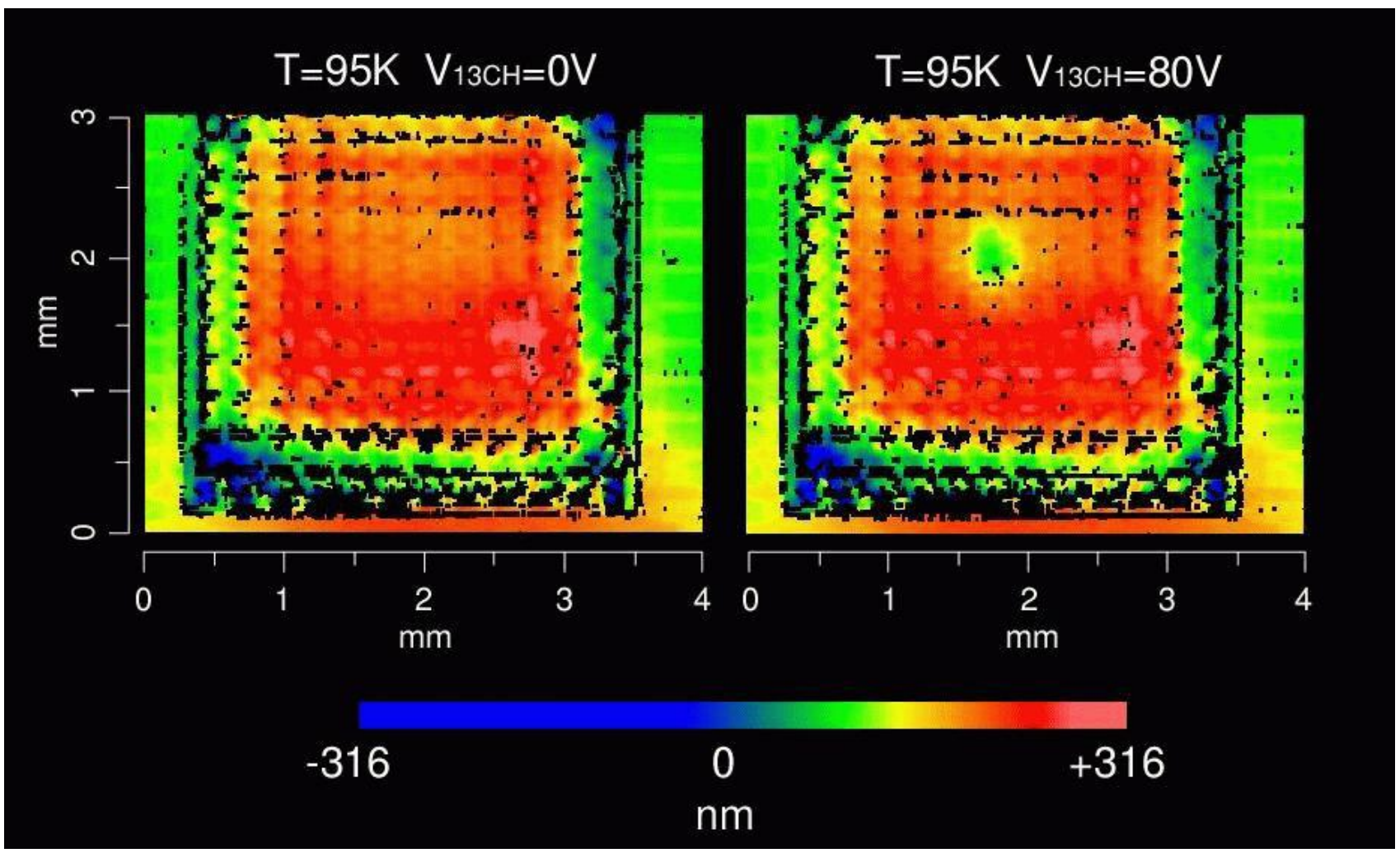

Figure 7: 3D surface data obtained from the Interferometer. All data were obtained by measurements made through the window of the vacuum cryostat. Left: surface without applied voltage at $95 \mathrm{~K}$. Right: surface with $80 \mathrm{~V}$ on the 13 th $\mathrm{CH}$ at $95 \mathrm{~K}$.

\subsection{Cryogenic tip-tilt mirror}

The SPICA telescope is influenced by mechanical vibration caused by cryo-coolers and other instruments. Such vibration corresponds to telescope pointing jitter of $\sim \operatorname{arcsec}$, which is not acceptable for the coronagraphic instrument or other MIR instruments of SPICA. So, we are developing cryogenic tip-tilt mirrors to compensate for the pointing jitter. Some prototypes of the cryogenic tip-tilt mirror have been manufactured in collaboration with a group at the Institute for Astronomy, University of Tokyo, working on ground-based astronomy, in which a cryogenic chopping mirror was developed and tested in a MIR camera, MAX 38. Figure 8 shows a prototype for one-axis chopping by a piezoelectric actuator. More detail is shown in Nakamura et al.(2008, this conference) ${ }^{[40]}$.

\section{SUMMARY}

SPICA is a next generation infrared space-borne telescope mission following AKARI. We are developing a MIR coronagraph for SPICA. A coronagraph with a binary shaped pupil, and a coronagraph with PIAA are being considered as candidates for the baseline solution and the advanced solution for SPICA, respectively. Laboratory demonstrations of both coronagraphs were performed with a visible laser in standard atmospheric conditions, and the requirement of the contrast for the SPICA coronagraph, better than $10^{-6}$, was satisfied. We are also developing cryogenic active optics. Actuation of a prototype MEMS DM was confirmed at cryogenic temperatures in a cryostat by liquid nitrogen. A cryogenic experiment employing a piezo-actuator for a tip-tilt mirror is on-going. 


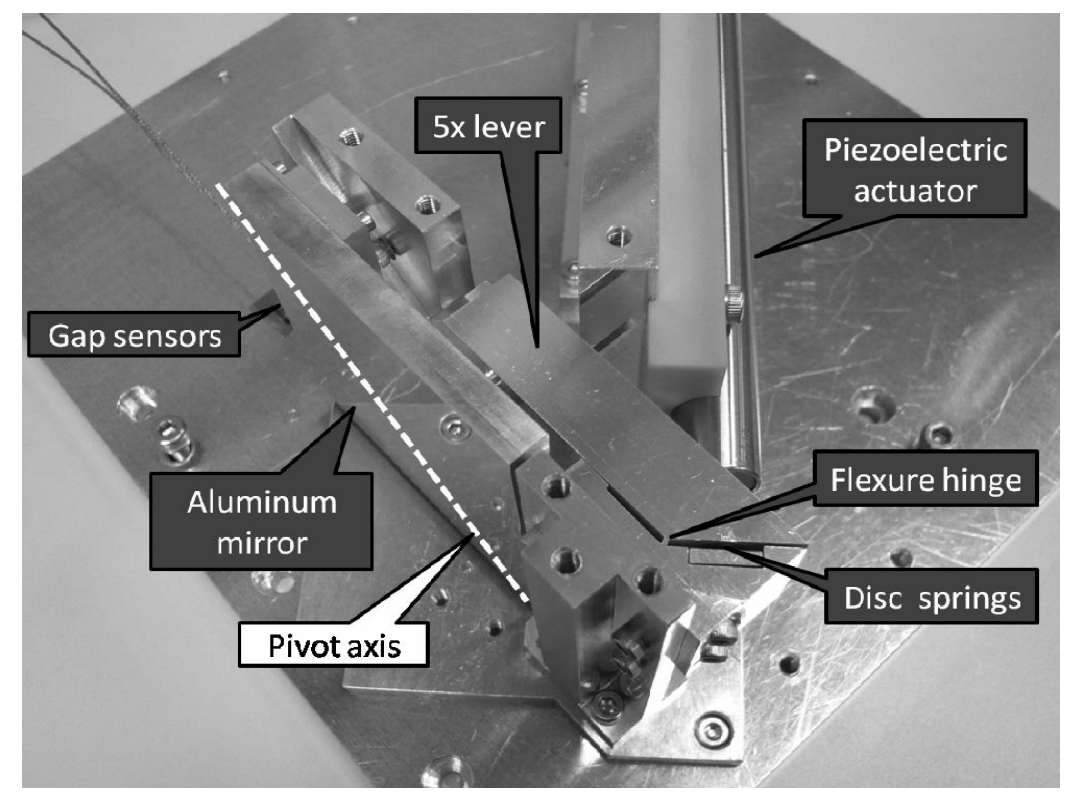

Figure. 8. Internal structure of a prototype of a cryogenic tip-tilt mirror system. The 6-cm square plane mirror is driven by a squarewave oscillation in axial rotation by a piezoelectric actuator. More detail is shown in Nakamura et al. (2008, this conference)

\section{ACKNOWLEGEMENT}

We are grateful for the kind support by T. Mitsui, M. Yokota, N. Okada, T. Nishino, T. Fukuda, K. Kaneko at the Advanced Technology Center of the National Astronomical Observatory, Japan. We would like to thank the Nanotechnology Research Institute of AIST of Japan. This work was supported in part by a grant from Japan Society for the Promotion of Science, and Ministry of Education, Culture, Sports, Science and Technology of Japan.

\section{REFERENCES}

1. Mayor, M., \& Queloz, D, “A Jupiter-Mass Companion to a Solar-Type Star”, D., Nature, 378, 355, 1995

2. Henry, G., W., Marcy, G. W., Butler, R. P., Vogt, S. S., “A Transiting "51 Peg-like" Planet”, ApJL, 529, 41, 2000

3. Charbonneau, D., Brown, T. M., Latham, D. W., Mayor, M., "Detection of Planetary Transits Across a Sun-like Star", ApJL, 529, 45, 2000

4. Traub, W. A., Jucks, K. W., “A Possible Aeronomy of Extrasolar Terrestrial Planets”, astro-ph/0205369, 2002

5. Lyot, B., "The study of the solar corona and prominences without eclipses", MNRAS., 99, 580, 1939

6. Nakagawa, T. and SPICA working goroup, "SPICA: space ingrared telescope for cosmology and astrophysics", $A d v$. Sp. Res., 34, 645, 2004

7. Onaka, T. and Nakagawa, T., "SPICA: a 3.5 meter space infrared telescope for mid-and far-infrared astronomy", Adv. Sp. Res., 36, 1123, 2005

8. Murakami, H., "ASTRO-F Infrared Sky Survey mission”, Proc. of SPIE, 5487, 330, 2004

9. K. Enya, L. Abe, S. Tanaka, K. Haze, M. Venet, T. Nakagawa, H. Kataza, M. Tamura, J. Nishikawa, N. Murakami, K. Fujita, Y. Itoh, O. Guyon, E. A. Pluzhnik, T. Wakayama, T. Sato, N. Nakagiri, SPICA working group, "Coronagraph Project with the SPICA Mission", Proc. of SPIE, 6693, 66930I, 2007

10. Abe, L., Enya, K., Tanaka, S., Nakagawa, T., Kataza, H., Tamura, M., Guyon, O., "Detecting extra-solar planets with the Japanese $3.5 \mathrm{~m}$ SPICA space telescope ”, Comptes Rendus Pysique, Optical Techniques for Direct Imaging of Exoplanets, 8, 374, 2007

11. Tamura, M., Abe, L. and Subaru HICHAO/SPICA coronagraph/JTPF teams, "Direct Explorations of Exoplanets with the Subaru Telescope and Beyond", Proc. of IAUC 200, 323, 2005 
12. Enya, K., Nakagawa. T., Kataza. H., Kaneda. H., Yui Y., Y., Tamura, M., Abe, L., Onaka, T., Takahashi, H. and the SPICA team, "Cryogenic infrared optics for the SPICA coronagraph", Proc. of IAUC 200, 467, 2005

13. Traub, W., A. et al., "TPF-C: status and recent progress", Proc. of SPIE, 6265, 26, 2006

14. Beichman, C., Lawson, P., Lay, O., Ahmed, A., Unwin, S., Johnston, K., "Status of the terrestrial planet finder interferometer (TPF-I)", Proc. of SPIE, 6268, 25, 2006

15. Leger, A., Mariotti, J. M., Mennesson, B., Ollivier, M., Puget, J. L., Rouan, D., Schneider, J., "The DARWIN Project", A\&SS, 241, 135, 1996

16. Cash, W., "Detection of Earth-like planets around nearby stars using a petal-shaped occulter", Nature, 422, 51, 2006

17. Sabelhaus, P. A. and Decker, J., "AN overview of the James Webb Space Telescope (JWST) project", Proc. of SPIE, 5487, 550, 20042004

18. Jacquinot, P., Roizen-Dossier, B., Prog. Opt., 3, 29, 1964

19. Spergel, D. N., "A New Pupil for Detecting Extrasolar Planets", astro-ph/0101142, 2001

20. Kasdin, N. J., Belikov, R., Beall, J., Vanderbei, R. J., Littman, M. G., Carr, M., Give'on, A., "Shaped pupil coronagraphs for planet finding: optimization, manufacturing, and experimental results", Proc. of SPIE, 5905, 128, 2005

21. Kasdin, N. J., Vanderbei, R. J., Littman, M. G. and Spergel, D. N., "Optimal one-dimensional apodizations and shaped pupils for planet finding coronagraphy", Appl. Opt., 44, 1117, 2005

22. Vanderbei, R. J., Spergel, D. N., \& Kasdin, N. J., "Spiderweb Masks for High-Contrast Imaging", ApJ, 590, 593603, 2003

23. Vanderbei, R. J., Kasdin, N. J. and Spergel, D. N., "Checkerboard-Mask Coronagraphs for High-Contrast Imaging", ApJ, 615, 555, 2004

24. Belikov, R., Give'On, A., Pueyo, L., Kasdin, J., Balasubramanian, K., Kern, B., Kuhnert, A., Shaklan, S., Trauger, T., "Laboratory Results in High Contrast Imaging with the Shaped Pupil Coronagraph", Proc. of the conference In the Spirit of Bernard Lyot, 2007

25. Tanaka, S., Enya, K., Nakagawa, T., Kataza, H. and Abe, L., "Binary-Shaped Pupil Coronagraph for High-Contrast Imaging Using a Space Telescope with Central Obstruction" ,PASJ, 58, 627, 2006

26. Enya, K., Tanaka, S., Abe, L., Nakagawa, T., "Laboratory experiment of checkerboard pupil mask coronagraph", $A \& A, \mathbf{4 6 1}, 783,2007$

27. Enya, K., Abe, L., Tanaka, S., Nakagawa, T., Haze, K.; Sato, T., Wakayama, T., "High contrast experiment of an AO-free coronagraph with a checkerboard pupil mask", $A \& A, \mathbf{4 8 0}, 899,2008$

28. Vanderbei, R., J., “Optimization Methods \& Software”, 12, 485, 1999

29. Guyon, O., "Phase-induced amplitude apodization of telescope pupils for extrasolar terrestrial planet imaging", $A \& A, \mathbf{4 0 4}, 379,2003$

30. Traub, W. A., Vanderbei, R. J., "Two-Mirror Apodization for High-Contrast Imaging”, ApJ, 599, 695, 2003

31. Pluzhnik, E. A., Guyon, O., Ridgway, S. T., Martinache, F., Woodruff, R. A., Blain, C., Galicher, R., "Exoplanet Imaging with a Phase-induced Amplitude Apodization Coronograph. II. Performance", ApJ, 644, 1246, 2006

32. Tanaka, S., Guyon, O., Pluzhnik, E. A., Abe, L., Enya, K., Nakagawa, T., "Laboratory Demonstration of the PIAA/Binary-Mask Hybrid Coronagraph", Proc. of the conference In the Spirit of Bernard Lyot, 2007

33. Aime, C., Soummer, R., "Multiple-stage apodized pupil Lyot coronagraph for high-contrast imaging", Proc. of SPIE, 5490, 456, 2004

34. L. Abe, M. Venet, K. Enya, H. Kataza, T. Nakagawa, M. Tamura, "Multi-Stage Apodized Pupil Lyot Coronagraph Experimental results", Proc. of SPIE (this conference), 2008

35. Venet, M., "Laboratory experiment of Prolate Apodized Lyot Coronagraph (PALC) for SPICA mission", master thesis, 2005

36. Dyson, H., Sharples, R. M., Dipper, N. A., Vdovin, G., "Cryogenic wavefront correction using membrane deformable mirrors", Optics Express, 8, 17, 2001

37. Mulvihill, M. L., Roche, M. E., Cavaco, J. L., Shawgo, R. J., Chaudhry, Z. A., Ealey, M. A., "Cryogenic deformable mirror technology development", Proc. of SPIE, 5172, 60, 2003

38. Bifano, T. G., Krishnamoorthy, R., Dorton, J. K., Perreault, J., Vandelli, N., Horenstein, M. N., Castanon, D. A., "Continuous-membrane surface-micromachined silicon deformable mirror", Opt. Eng., 36, 1354, 1997

39. J. B. Stewart, T. G. Bifano, S. Cornelissen, P. Bierden, B. M. Levine, "Design and development of a 331-segment tip-tilt-piston mirror array for space-based adaptive optics", Sensor and Actuators A: Physical, 138, 230, 2007

40. Nakamura, T., Miyata, T., Sako, S., Onaka, T., Enya, K., Kataza, H., Takahashi, H., Obuchi, Y., "Cold Chopper System for Mid-infrared Instruments", Proc. of SPIE (this conference), 2008 